\title{
X-linked osteoporosis with fractures
}

INSERM

\section{Source}

INSERM. (1999). Orphanet: an online rare disease and orphan drug data base. $\underline{X \text {-linked }}$ osteoporosis with fractures. ORPHA:391330

X-linked osteoporosis with fractures is a rare, genetic, primary bone dysplasia with decreased bone density disorder characterized by childhood-onset osteoporosis associated with recurrent, multiple, osteoporotic, long bone fractures and/or vertebral compression fractures, significant height loss in adulthood, low bone mineral density scores, and otherwise no other abnormalities. Heterozyg ote females may be unaffected or have a milder phenotype. 